Article

\title{
Temperature and Pulse-Energy Range Suitable for Femtosecond Pulse Transmission in Si Nanowire Waveguide
}

\author{
Xiaochun Wang ${ }^{1}\left(\mathbb{D}\right.$, Meicheng Fu ${ }^{1}$, Heng Yang ${ }^{2}$, Jiali Liao ${ }^{3}$ and Xiujian $\mathrm{Li}^{1, *}$ \\ 1 Department of Physics, College of Liberal Arts and Sciences, National University of Defense Technology, \\ Changsha 410073, China; xiaochunwang01@163.com (X.W.); fumeicheng10@nudt.edu.cn (M.F.) \\ 2 College of Information and Communication, National University of Defense Technology, Changsha 410073, \\ China; hengy@yeah.net \\ 3 School of Physics and Optoelectronic Engineering, Xidian University, Xi'an 710071, China; \\ liaojiali@xidian.edu.cn \\ * Correspondence: xjli@nudt.edu.cn
}

Received: 28 October 2020; Accepted: 20 November 2020; Published: 26 November 2020

\begin{abstract}
We experimentally measured the femtosecond pulse transmission through a silicon-oninsulator (SOI) nanowire waveguide under different temperatures and input pulse energy with a cross-correlation frequency-resolved optical gating (XFROG) measurement setup. The experimental results demonstrated that the temperature and pulse energy dependence of the Si photonic nanowire waveguide (SPNW) is interesting rather than just monotonous or linear, and that the suitable temperature and pulse-energy range is as suggested in this experiment, which will be valuable for analyzing the practical design of the operating regimes and the fine dispersion engineering of various ultrafast photonic applications based on the SPNWs. The research results will contribute to developing the SPNWs with photonic elements and networks compatible with mature complementary metal-oxide-semiconductors (CMOS).
\end{abstract}

Keywords: Si photonic nanowire waveguides; blue shift; pulse acceleration; pulse broadening; temperature

\section{Introduction}

With the ability to tightly confine optical modes [1] and its compatibility with mature complementary metal-oxide-semiconductors (CMOS) technologies [2], silicon photonic nanowire waveguides (SPNWs) have been extensively studied [3-5] for various photonic technologies, such as temporal soliton-effects compression [6-8], four-wave mixing [9-11], optical switching [12], and all-optical modulation [13,14]. As a basic platform for many photonic technologies and elements, such as silicon photonic crystal waveguide [15,16] and micro-rings [17-20], which can be widely used in many applications [15-18,21], the SPNWs' flexibility for applications should be analyzed, especially their environmental temperature adaptability $[19,20,22,23]$. The thermo-optic coefficient (TOC) of $1.86 \times 10^{-4} \mathrm{~K}^{-1}$ is large enough to show that the device is extremely sensitive to temperature [23,24]. The thermo-optic coefficient, nonlinear refractive index [25], Kerr nonlinearity, and two-photon absorption [26,27] have been researched in many materials such as Si, Si3N4, and SiOx [26-28]. The Mach-Zehnder interferometer (MZI)-based temperature sensor with hybrid waveguides on silicon [4,23,29], the all-silicon interferometer [30], the precision distance measurement [27], and so on have been designed and applied in many optical, chemical, or biological processes.

For further applications in optical communications, optical signal processing, and microwave photonics, if the long pulse, i.e., the pulse duration, are not shorter than $1 \mathrm{~ns}$, they will be displaced 
by ultra-short pulses such as femtosecond pulses and solitons [31,32]. Therefore, besides under room temperature, the propagation of the femtosecond pulses in the SPNW under a suitable range temperature around room temperature with a suitable range pulse energy is necessary for the dispersion engineering photonic elements based on the SPNWs, which have to consider the Group velocity dispersion (GVD) and various nonlinear factors that affect the pulses evolution, including self-phase modulation (SPM), two-photon absorption (TPA), free carrier absorption (FCA) and free carrier dispersion (FCD), and so on.

With the sensitive cross-correlation frequency-resolved optical gating (XFROG) measurement setup $[33,34]$, we measured the picojoule femtosecond pulse propagation in a silicon-on-insulator (SOI) nanowire waveguide under different temperatures around room temperature for various inputs of pulse energy, so as to find the suitable temperature and pulse energy range for future applications. Variations of the temporal pulse profile and the wavelength profile under different temperatures demonstrate that the temperature and pulse energy dependence of the SPNW is not monotonous and linear, which will be valuable for analysis of the design of operating regimes and the fine dispersion engineering of various ultrafast photonic elements based on the SPNWs for optical communication, optical computing, and microwave photonics.

\section{Measurement Details}

The SPNW for our experiment is fabricated on a SOI wafer, which has a profile of $250 \mathrm{~nm}-\mathrm{Si}$, $3 \mu \mathrm{m}-\mathrm{SiO} 2$, and $500 \mu \mathrm{m}-\mathrm{Si}$. The SPNW is designed as a cross-section of $\mathrm{A} 0=450 \mathrm{~nm} \times 250 \mathrm{~nm}$ and has a length of $\mathrm{L}=4 \mathrm{~mm}$, and is further covered by a 3- $\mu \mathrm{m}$ top silicon oxide layer. Furthermore, mode adapters are integrated into the waveguide input and output coupling facets to reduce the coupling loss to less than $3 \mathrm{~dB}$ per facet and suppress any facet Fabry-Perot oscillations [31]. Figure 1 shows the scanning electron microscope (SEM) image and 3-D structural detail chart of the SPNW.

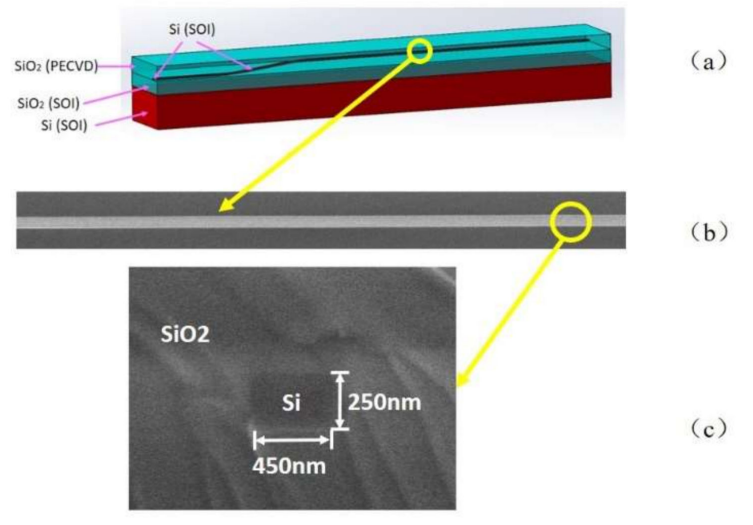

Figure 1. The structural diagram of the Si photonic nanowire waveguide (SPNW). (a) 3-D structure detail chart with top right 45-degree view. (b) Top view SEM of the partial SPNW before overlaying the top silicon oxide layer. (c) SEM of the right view after overlaying the top silicon oxide layer.

The temporal and spectral profiles of the pulses were measured by the experimental setup with an attojoule-sensitive XFROG as shown in Figure 2. The mode-locked femtosecond pulse fiber laser is customized by CSA IoP, which can output femtosecond pulses with pulse duration FWHM of $150 \mathrm{fs}$ and wavelength FWHM of $60 \mathrm{~nm}$. To measure dynamics of ultra-short femtosecond pulses, we constructed a sensitive second-harmonic XFROG setup based on a BBO crystal (1-mm thickness, provided by CASTECH INC. CHINA) and a Horiba JY FHR1000 spectrometer with a SYMPHONY II UVCCD-1024 $\times 256$-BIDD detector, which enabled the minimum detected pulse energy to be down to about $500 \mathrm{aJ}$, and the spectral resolution to be better than $0.1 \mathrm{~nm}$. There was also a programmable, finely motorized translation stage, THORLABS Z825, which enabled the finely moving controlled retro reflector mirror to provide temporal resolution down to less than $1 \mathrm{fs}$ for the measurement setup. 
The input pulse energy can be finely controlled by a set of half-wave plates and linear polarizers mounted on a motorized rotation stage. In order to finely control the temperature of the SPNW chip for measurement (DUT in Figure 2), the SPNW chip was bonded on a thermoelectric cooler (TEC) driven by a TEC temperature controller (THORLABS TED4015), which can provide a temperature control range of -55 to $150{ }^{\circ} \mathrm{C}$ and a temperature control resolution better than $0.002{ }^{\circ} \mathrm{C}$. The output pulse will also be measured by an optical spectrum analyzer (Anritsu MS96A), which has a measurement wavelength resolution of $0.02 \mathrm{~nm}$. A flip mirror will switch the optical path for OSA and XFROG measurements.

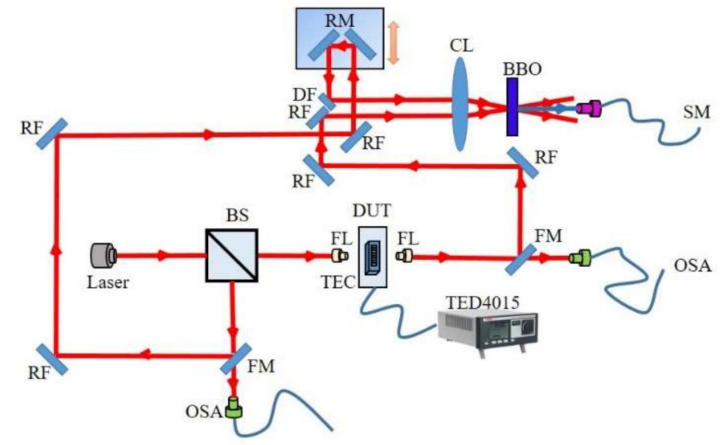

Figure 2. The experimental setup with cross-correlation frequency-resolved optical gating (XFROG). BS: Beam splitter; FL: Focusing lens; TEC: Thermo electric cooler; DUT: SPNW chip; TED 4015: TEC temperature controller; FM: Flip mirror; RF: Reflector; OSA: optical spectrum analyzer; DM: D-shaped mirror; RM: Retro reflector mirror; CL: Bi-convex lens; BBO: barium borate crystal; SM: Spectrometer with charge-coupled detector (CCD).

\section{Experimental Results}

The experimental results retrieved from the data measured by XFROG and OSA are shown in Figures 4-9, where the data measured by OSA were calibrated during the retrieval of data in the experiments, the pulse energy was chosen discretely, i.e., 1.1, 2.7, 5.3, 13.3, 26.7, and $35.2 \mathrm{pJ}$, respectively, and the temperature was set to 12,25 , and $52.8^{\circ} \mathrm{C}$ for every pulse energy measurement. The central wavelength of the input femtosecond pulse for measurements was $1550 \mathrm{~nm}$, the temporal and spectral profiles of which are shown in Figure 3. The input pulse contains double temporal peaks (as shown in Figure 3a), with FWHM $113.4 \mathrm{fs}$ and $89.3 \mathrm{fs}$, and a hollow down to 0.4 existing in the normalized wavelength profile to perform double spectral peaks, which will help us to analyze the dynamics further.

(a)

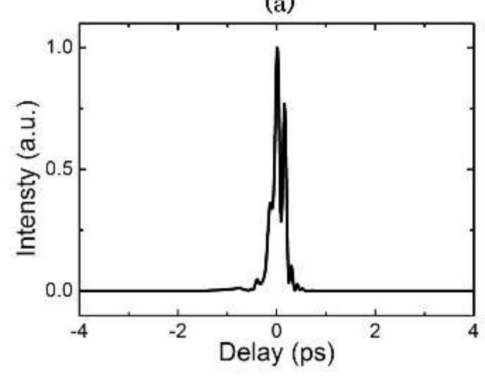

(b)

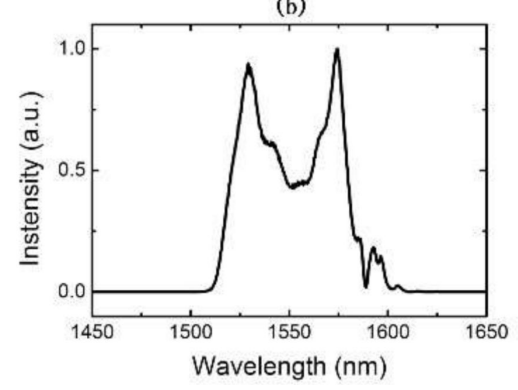

Figure 3. Temporal intensity profiles (a) and spectral intensity profiles (b) of the input pulses.

Figures 4 and 5 illustrate that the pulse will show pulse acceleration, splitting, and trailing, which become more obvious when the input pulse energy increases at room temperature $\left(25^{\circ} \mathrm{C}\right)$ and low temperature $\left(12{ }^{\circ} \mathrm{C}\right)$. Furthermore, the front sub-pulse shows pulse acceleration with low input pulse energies, and shows broadening and multiple tiny spikes splitting with higher input pulse energies. Furthermore, from the spectral lines, we found that the output pulse had a sharp peak in a long wavelength with higher input pulse energy. 


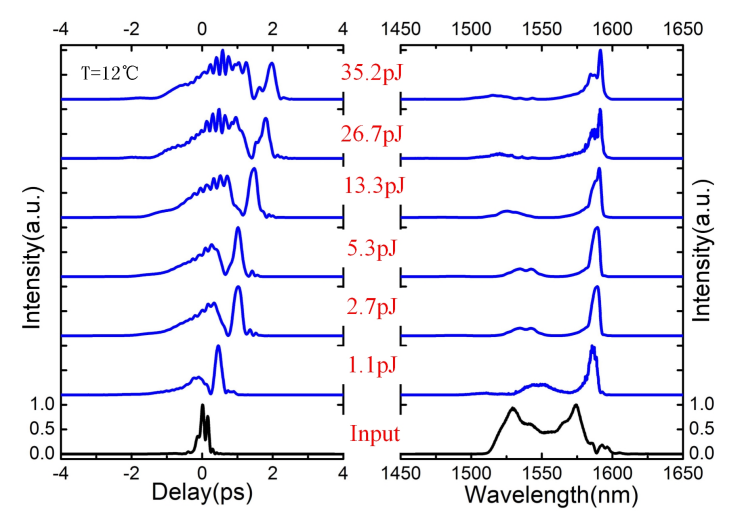

Figure 4. Temporal and spectral intensity profiles of the output pulses retrieved from the XFROG measurements for the coupled pulse energies that increased from $1.1 \mathrm{pJ}$ to $35.2 \mathrm{pJ}$ at the temperature of $12{ }^{\circ} \mathrm{C}$.

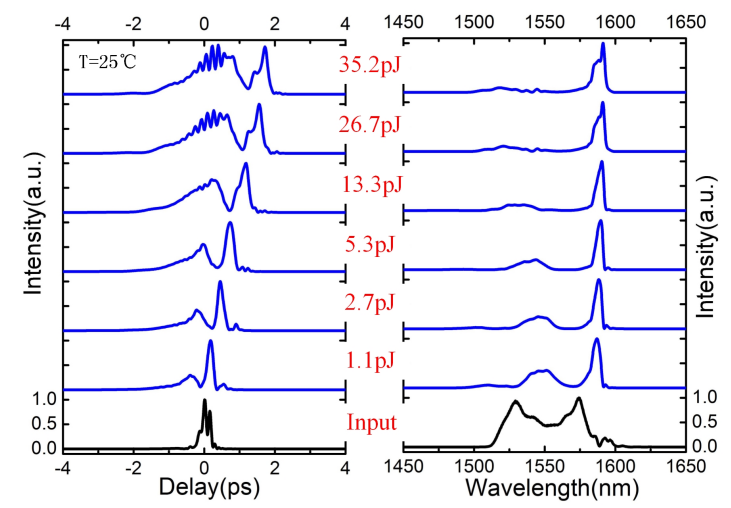

Figure 5. Temporal and spectral intensity profiles of the output pulses retrieved from the XFROG measurements for the coupled pulse energies that increased from $1.1 \mathrm{pJ}$ to $35.2 \mathrm{pJ}$ at the temperature of $25^{\circ} \mathrm{C}$.

As shown in Figure 6, we found that, under high temperature and low pulse energy, the pulse will have a temporal profile where the acceleration will dominate the transmission, and the corresponding blue shift is clearly noticeable in the spectral lines. With the increase of pulse energy, the relative intensity of long wavelength sub-pulse increases, while the blue shift sub-pulse gradually weakens, which was the result of the output of red shift spectral peak and trailing temporal pulse.

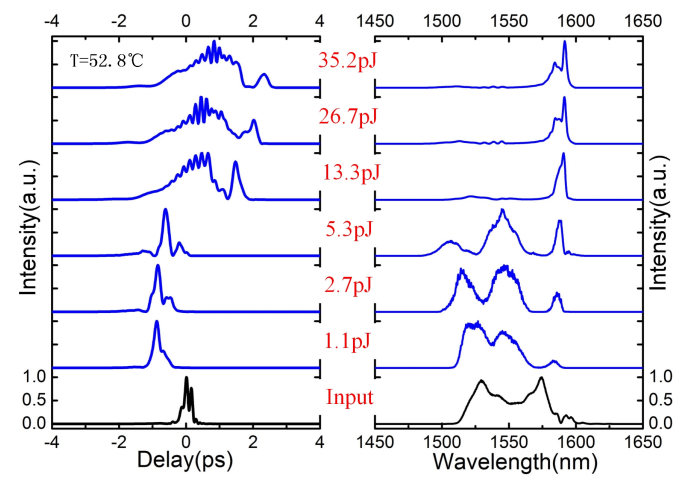

Figure 6. Temporal and spectral intensity profiles of the output pulses retrieved from the XFROG measurements for the coupled pulse energies that increased from $1.1 \mathrm{pJ}$ to $35.2 \mathrm{pJ}$ at the temperature of $52.8^{\circ} \mathrm{C}$. 


\section{Analysis and Discussion}

According to the above experimental results, the femtosecond pulse will undergo a much different propagation in the waveguide for different temperatures and input pulse energy, which may owe to the thermal characteristics and the free carrier effects of the silicon. Silicon is temperature sensitive, with a large TOC of about $1.86 \times 10^{-4} \mathrm{~K}^{-1}$. There may be many kinds of nonlinear effects with the femtosecond pulse propagation in the SPNW, such as the SPM, TPA, FCA, FCD, and Kerr nonlinearity. The main phenomena are pulse acceleration, blue shift and red shift, pulse broadening, and pulse compression with different temperatures and inputs of pulse energy, as shown in Figures 7-12. When only the SPM and GVD are considered, the produced chirp just affects the blue shift of the pulse and is symmetrical to the front and back edges of the pulse [35]. At the same time, the TPA is proportional to the light intensity in the SOI waveguide, which produces a large number of free carriers, and then the FCD and FCA change. Kerr nonlinearity is based on the second order refractive index, and the temperature changes the index of the SPNW. The experiment above changed the inputs of pulse energy and the temperatures of the SPNW, and then separated the effect of the temperature on the refractive index as the thermo-optic effect (TOE) [36] in order to discuss the influence of temperature of the pulse transmission in the SPNW more clearly.
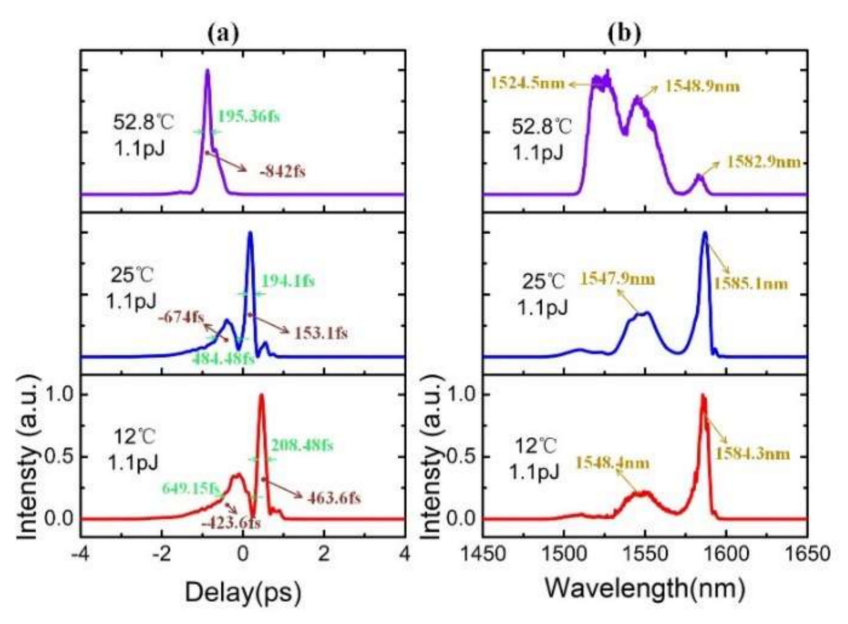

Figure 7. Temporal intensity profiles (a) and spectral intensity profiles (b) of the output pulses retrieved from the XFROG measurements for $1.1 \mathrm{pJ}$ input pulse energy at temperatures of $12{ }^{\circ} \mathrm{C}, 25^{\circ} \mathrm{C}$, and $52.8^{\circ} \mathrm{C}$.
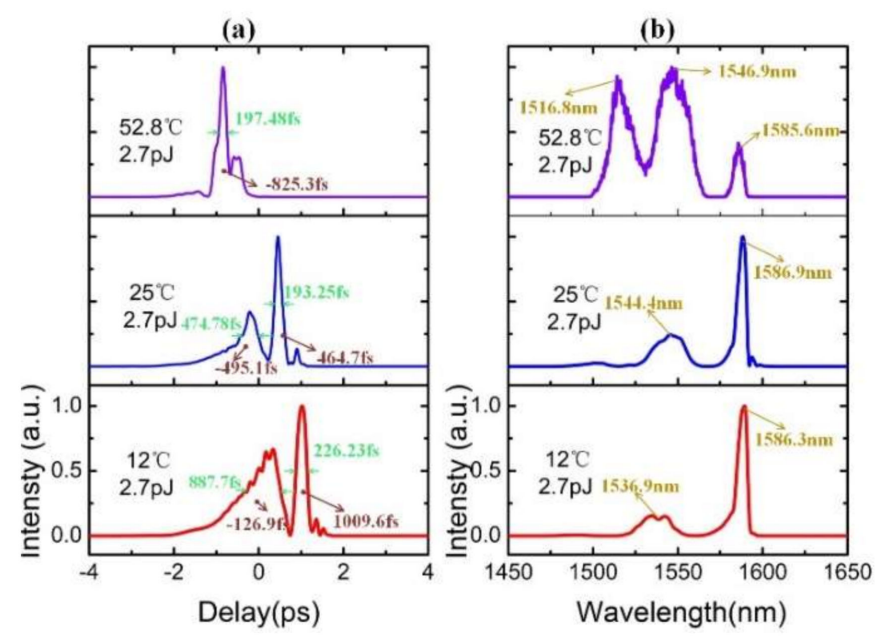

Figure 8. Temporal intensity profiles (a) and spectral intensity profiles (b) of the output pulses retrieved from the XFROG measurements for $2.7 \mathrm{pJ}$ input pulse energy at temperatures of $12{ }^{\circ} \mathrm{C}, 25^{\circ} \mathrm{C}$, and $52.8^{\circ} \mathrm{C}$. 

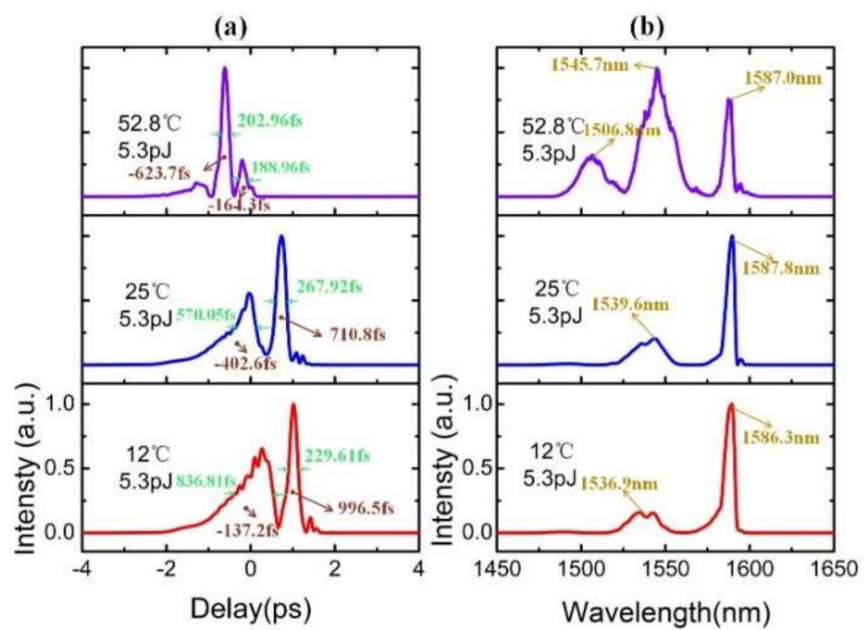

Figure 9. Temporal intensity profiles (a) and spectral intensity profiles (b) of the output pulses retrieved from the XFROG measurements for $5.3 \mathrm{pJ}$ input pulse energy at temperatures of $12{ }^{\circ} \mathrm{C}, 25^{\circ} \mathrm{C}$, and $52.8^{\circ} \mathrm{C}$.
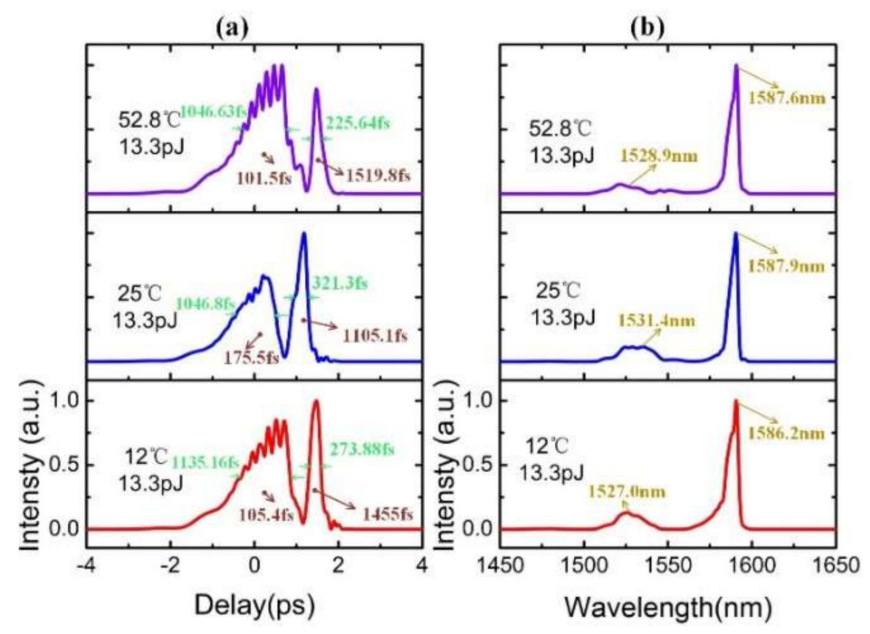

Figure 10. Temporal intensity profiles (a) and spectral intensity profiles (b) of the output pulses retrieved from the XFROG measurements for $13.3 \mathrm{pJ}$ input pulse energy at temperatures of $12{ }^{\circ} \mathrm{C}, 25^{\circ} \mathrm{C}$, and $52.8^{\circ} \mathrm{C}$.
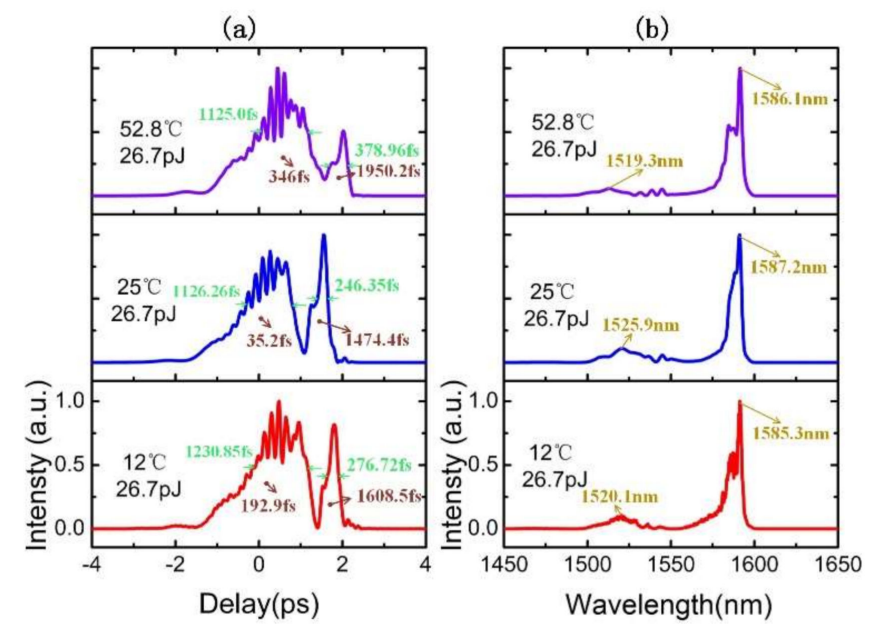

Figure 11. Temporal intensity profiles (a) and spectral intensity profiles (b) of the output pulses retrieved from the XFROG measurements for $26.7 \mathrm{pJ}$ input pulse energy at different temperatures of $12{ }^{\circ} \mathrm{C}, 25^{\circ} \mathrm{C}$, and $52.8^{\circ} \mathrm{C}$. 
(a)

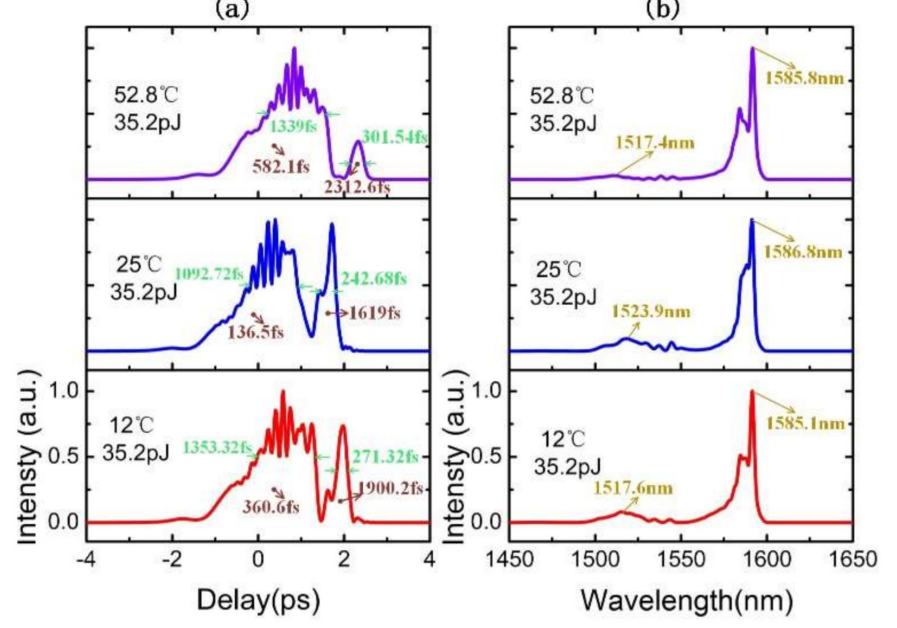

Figure 12. Temporal intensity profiles (a) and spectral intensity profiles (b) of the output pulses retrieved from the XFROG measurements for $35.2 \mathrm{pJ}$ input pulse energy at temperatures of $12{ }^{\circ} \mathrm{C}, 25^{\circ} \mathrm{C}$, and $52.8^{\circ} \mathrm{C}$.

According to Figures 7-9, in the case of low pulse energy, with the increase of temperature, the blue shift will be more remarkable and the pulse acceleration will be more developed, which is considered to be mainly induced by the FCA in silicon nanowire waveguides [35]. We also can find that, in the case of low pulse energy, the femtosecond pulse will be split into two temporal sub-peaks at low temperature, while it will be split into three spectral sub-peaks at high temperature, i.e., $1520 \mathrm{~nm}$, $1540 \mathrm{~nm}$, and $1584 \mathrm{~nm}$ at $52.8^{\circ} \mathrm{C}$, which also confirms that the FCA effect has taken place, and that the red shift was simultaneously induced by the dispersive wave.

For the high pulse energy cases, as shown in Figures 10-12, the temporal pulse split and pulse broadening was remarkable, while the spectral energy was concentrated on the red-shift wavelength, and the blue-shift part weakened and even disappeared gradually. Furthermore, the pulses decelerated rather than accelerated, which could be the result of the weakening and disappearing of the blue-shift part. Therefore, the pulse propagation will not mainly depend on the temperature, which confirms that the FCA will dominate the propagation for high pulse energy cases, in which strong pulses will produce high concentration free carriers. Thus, for the high pulse energy cases, the output pulse is similar and independent of temperature and input energy, which leads to output pulses with narrow spectral bands.

The data for Figures 7-12 are listed in Tables 1-3. The output sharp pulses perform enhanced acceleration and pulse compression with the increase of temperature when there is a higher input of pulse energy. Relative to $12{ }^{\circ} \mathrm{C}$, the output of sharp pulse acceleration is $283.5 \mathrm{fs}$ at $25^{\circ} \mathrm{C}$ and $1278.6 \mathrm{fs}$ at $52.8^{\circ} \mathrm{C}$ for an input energy of $1.1 \mathrm{pJ}, 554.9 \mathrm{fs}$ at $25^{\circ} \mathrm{C}$ and $1834.9 \mathrm{fs}$ at $52.8^{\circ} \mathrm{C}$ for an input energy of $2.7 \mathrm{pJ}$, and $285.7 \mathrm{fs}$ at $25^{\circ} \mathrm{C}$ and $1620.2 \mathrm{fs}$ at $52.8^{\circ} \mathrm{C}$ for an input energy of $5.3 \mathrm{pJ}$, as shown in Table 3 .

Table 1. Pulse duration of the output pulses measured by XFROG (FWHM).

\begin{tabular}{|c|c|c|c|c|c|c|c|c|c|}
\hline \multirow{3}{*}{ Pulse Energy (pJ) } & \multicolumn{3}{|c|}{$12.0^{\circ} \mathrm{C}$} & \multicolumn{3}{|c|}{$25.0^{\circ} \mathrm{C}$} & \multicolumn{3}{|c|}{$52.8^{\circ} \mathrm{C}$} \\
\hline & \multicolumn{3}{|c|}{ FWHM (fs) } & \multicolumn{3}{|c|}{ FWHM (fs) } & \multicolumn{3}{|c|}{ FWHM (fs) } \\
\hline & Ahead & Back & Total & Ahead & Back & Total & Ahead & Back & Total \\
\hline 1.1 & 649.15 & 208.48 & 208.48 & 484.48 & 194.10 & 194.10 & 195.36 & $\mathrm{NON}$ & 195.36 \\
\hline 2.7 & 887.7 & 226.23 & 226.23 & 474.78 & 193.25 & 193.25 & 197.48 & NON & 197.48 \\
\hline 5.3 & 836.81 & 229.61 & 229.61 & 570.05 & 267.92 & 267.92 & 202.96 & 188.96 & 202.96 \\
\hline 13.3 & 1135.16 & 273.88 & 273.88 & 1046.80 & 321.30 & 321.30 & 1046.63 & 225.64 & 1046.63 \\
\hline 26.7 & 1230.85 & 276.72 & 1230.85 & 1126.26 & 246.35 & 246.35 & 1125.00 & 378.96 & 1125.00 \\
\hline 35.2 & 1353.32 & 271.32 & 1353.32 & 1092.72 & 242.68 & 242.68 & 1339.00 & 301.54 & 1339.00 \\
\hline
\end{tabular}


Table 2. Spectral pulse centroids of the output pulses.

\begin{tabular}{cccccccccc}
\hline & \multicolumn{3}{c}{$\mathbf{1 2 . 0}{ }^{\circ} \mathbf{C}$} & \multicolumn{3}{c}{$\mathbf{2 5 . 0}{ }^{\circ} \mathbf{C}$} & \multicolumn{3}{c}{$\mathbf{5 2 . 8}{ }^{\circ} \mathbf{C}$} \\
\cline { 2 - 10 } Pulse Energy (pJ) Centroid (nm) & \multicolumn{3}{c}{ Centroid (nm) } \\
& Left & Centroid (nm) & Right & Left & Center & Right & Left & Center & Right \\
\hline 1.1 & 1548.4 & NON & 1584.3 & 1547.9 & NON & 1585.1 & 1524.5 & 1548.9 & 1582.9 \\
2.7 & 1536.9 & NON & 1586.3 & 1544.4 & NON & 1586.9 & 1516.8 & 1546.9 & 1585.6 \\
5.3 & 1536.9 & NON & 1586.3 & 1539.6 & NON & 1587.8 & 1506.8 & 1545.7 & 1587.0 \\
13.3 & 1527.0 & NON & 1586.2 & 1531.4 & NON & 1587.9 & 1528.9 & NON & 1587.6 \\
26.7 & 1520.1 & NON & 1585.3 & 1525.9 & NON & 1587.2 & 1519.3 & NON & 1586.1 \\
35.2 & 1517.6 & NON & 1585.1 & 1523.9 & NON & 1586.8 & 1517.4 & NON & 1585.8 \\
\hline
\end{tabular}

Table 3. Temporal pulse centroids of the output pulses.

\begin{tabular}{cccccccccc}
\hline & \multicolumn{3}{c}{$\mathbf{1 2 . 0}{ }^{\circ} \mathbf{C}$} & \multicolumn{3}{c}{$\mathbf{2 5 . 0}{ }^{\circ} \mathbf{C}$} & \multicolumn{3}{c}{$\mathbf{5 2 . 8 ^ { \circ } \mathbf { C }}$} \\
\cline { 2 - 11 } Pulse Energy (pJ) & \multicolumn{3}{c}{\begin{tabular}{c} 
Centroid (fs) \\
\cline { 2 - 11 }
\end{tabular}} & Ahead & Back & Total & Ahead & Centroid (fs) & \multicolumn{3}{c}{ Centroid (fs) } \\
Back & Total & Ahead & Back & Total \\
\hline 1.1 & -423.6 & 463.6 & -14.1 & -674.0 & 153.1 & -199.0 & NON & NON & -842.0 \\
2.7 & -126.9 & 1009.6 & 218.9 & -495.1 & 464.7 & -60.1 & NON & NON & -825.3 \\
5.3 & -137.2 & 996.5 & 217.5 & -402.6 & 710.8 & 53.1 & -623.7 & -164.3 & -680.3 \\
13.3 & 105.4 & 1455.0 & 406.5 & 175.5 & 1105.1 & 224.7 & 101.5 & 1519.8 & 313.1 \\
26.7 & 192.9 & 1608.5 & 529.8 & 35.2 & 1474.4 & 384.9 & 346.0 & 1950.2 & 551.9 \\
35.2 & 360.6 & 1900.2 & 617.9 & 136.5 & 1619.0 & 454.0 & 582.1 & 2312.6 & 694.0 \\
\hline
\end{tabular}

As shown in Figure 13, the output pulse temporal centroids agree with the spectral centroids for different coupled input pulse energies at different temperatures. When the energy of input pulses increased, the TPA intensity increased, as did the enhancement of FCA and FCD, and the output pulses showed pulse deceleration and red shift. The same phenomenon was illustrated in Figure $13 \mathrm{c}, \mathrm{d}$. The FCA of SPNW for femtosecond pulses caused pulse deceleration, and the TOE caused the move in the opposite direction for the femtosecond pulse [36]. Further, Figure 13a shows that the TOE caused pulse acceleration and the effect was nonlinear. The FCD of SPNW for femtosecond pulse caused pulse red shift and the TOE caused the blue shift [36]. Figure 13b shows that the TOE caused pulse blue shift and the effect was nonlinear.

(a)

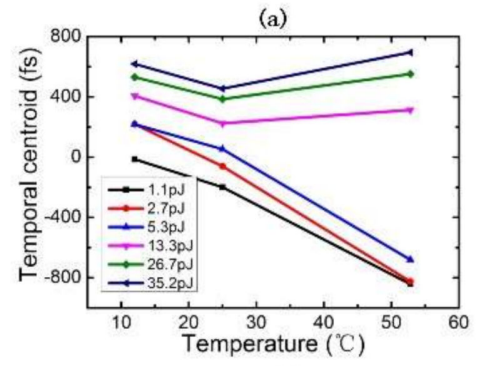

(c)

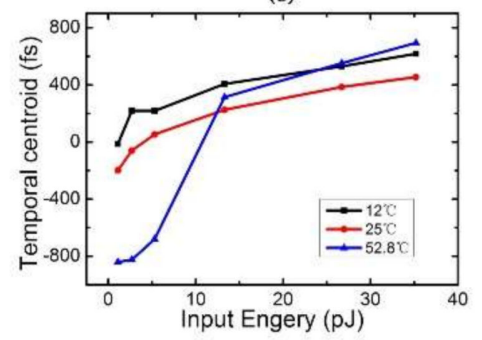

(b)

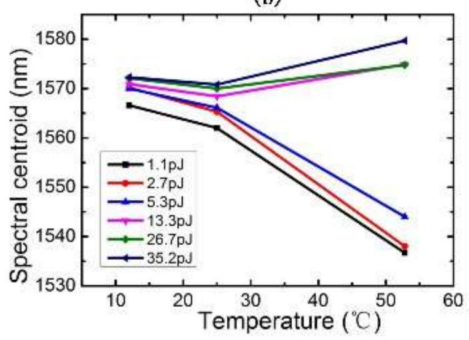

(d)

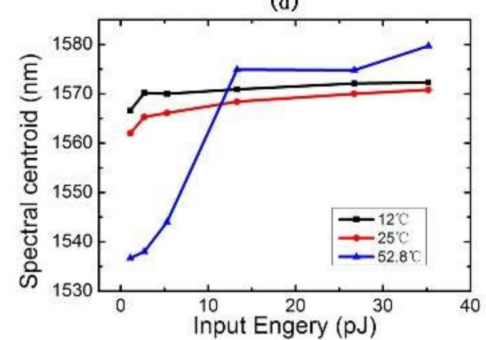

Figure 13. Temporal centroid $(\mathbf{a}, \mathbf{c})$ and spectral centroid $(\mathbf{b}, \mathbf{d})$ for different input pulses energies while the SPNW is at different temperatures.

As the silicon has a large temperature sensitivity due to the TOC, the indexes of the SPNW are influenced by TOC. The measurements indicate that the asymmetry of FCA and FCD is enhanced and 
that the nonlinear influence of TOE makes the relative intensity distribution of pulse energy change, which causes the change in pulse velocity and pulse acceleration. With the increasing temperature, the output pulses will be split and broadened to a great extent with high input pulse energy, as shown in Figures 10-12 and Table 1. The TPA and FCD affect pulse broadening and splitting [35], which may lead to multi sub-peaks such as the left, the center, and the right peaks as shown in Table 1, Figure 13b, and Table 2.

In brief, for low pulse energy inputs, we should make the waveguide work at low temperature to achieve major narrow spectral peaks and temporal peaks. While for high pulse energy input, the waveguide can work stably at a wide range of temperatures.

\section{Conclusions}

We have observed the femtosecond pulses transmit in SPNW with different input energies at different temperatures by experiments. The large thermo-optic coefficient (TOC) of silicon has high temperature sensitivity for the device controlled by temperature. The femtosecond pulses transmit nonlinear characteristics such as SPM, FCA, FCD, TPA, Kerr nonlinearity, and TOE in SPNW. TPA intensity is influenced by the input pulse energy and can influence the free carrier concentration. Thus, TPA intensity can influence FCA and FCD. The FCA of the SPNW for femtosecond pulses causes pulse deceleration. The FCD of the SPNW for femtosecond pulses causes pulse red shift. The TOE caused pulse acceleration and blue shift, and this influence is of nonlinear variation. The output pulses are characterized by acceleration, splitting, compression, and broadening for different input energies at various temperatures. These results provide basic references for researching SPNW for femtosecond pulse applications such as photonic networks. They also show that the SPNW exports single peak femtosecond pulses with dual-peak femtosecond input pulses and achieves pulse acceleration by adjusting the SPNW temperatures and the input pulse energies. The silicon nanowire waveguide will output long wavelength sharp pulses with small central wavelength disparity by controlling the temperature and input energy. For different inputs of pulse energy, we should make the waveguide work in corresponding suitable temperatures. The research results will contribute to the SPNWs' development for photonic elements and networks compatible with mature CMOS.

Author Contributions: Conceptualization, X.L.; and X.W.; validation, M.F.; investigation, J.L.; data curation, X.W.; writing-original draft preparation, X.W.; writing-review and editing, X.L.; supervision, H.Y.; All authors have read and agreed to the published version of the manuscript.

Funding: National Science Foundation of China (NSFC) (61070040, 61108089, 61205087, 61107005 62005207), Hunan Provincial Natural Science Foundation of China (805297133191), Natural Science Foundation of Shaanxi Province (2019JQ-648).

Acknowledgments: The authors acknowledge assistance from Ken Liu.

Conflicts of Interest: The authors declare no conflict of interest.

\section{References}

1. Almeida, V.R.; Barrios, C.A.; Panepucci, R.R.; Lipson. M. All-optical control of light on a silicon chip. Nature 2004, 431, 1081-1084. [CrossRef]

2. Dai, D.; Bowers, J.E. Novel concept for ultracompact polarization splitter-rotator based on silicon nanowires. Opt. Express 2011, 19, 10940-10949. [CrossRef]

3. Foster, M.A.; Turner, A.C.; Lipson, M.; Gaeta, A.L. Nonlinear optics in photonic nanowires. Opt. Express 2008, 16, 1300-1320. [CrossRef] [PubMed]

4. Velasco, A.V.; Cheben, P.; Bock, P.J.; Delâge, A.; Schmid, J.H.; Lapointe, J.; Janz, S.; Calvo, M.L.; Xu, D.; Czyk, M.A.F.; et al. High-resolution Fourier-transform spectrometer chip with microphotonic silicon spiral waveguides. Opt. Lett. 2013, 38, 706-708. [CrossRef] [PubMed]

5. Liu, K.; Xu, W.; Zhu, Z.H.; Ye, W.M.; Yuan, X.D.; Zeng, C. Wave propagation in deep-subwavelength mode waveguides. Opt. Lett. 2012, 37, 2826-2828. [CrossRef] [PubMed] 
6. Tan, D.T.H.; Sun, P.C.; Fainman, Y. Monolithic nonlinear pulse compressor on a silicon chip. Nat. Commun. 2010, 1, 116. [CrossRef]

7. Foster, M.A.; Gaeta, A.L.; Cao, Q.; Trebino, R. Soliton-effect compression of supercontinuum to few-cycle durations in photonic nanowires. Opt. Express 2005, 13, 6848-6855. [CrossRef]

8. Zhou, H.; Huang, S.; Li, X.; McMillan, J.F.; Zhang, C.; Wong, K.K.; Yu, M.; Lo, G.; Kwong, D.; Qiu, K.; et al. Real-time dynamics and cross-correlation gating spectroscopy of free-carrier Drude slow-light solitons. Light Sci. Appl. 2017, 6, P49-P56. [CrossRef]

9. Driscoll, J.B.; Ophir, N.; Grote, R.R.; Dadap, J.I.; Panoiu, N.C.; Bergman, K.; Osgood, R.M. Width-modulation of $\mathrm{Si}$ photonic wires for quasi-phase-matching of four-wave-mixing: Experimental and theoretical demonstration. Opt. Express 2012, 20, 9227-9242. [CrossRef]

10. McMillan, J.F.; Yu, M.; Kwong, D.-L.; Wong, C.W. Observations of four-wave mixing in slow-light silicon photonic crystal waveguides. Opt. Express 2010, 18, 15484-15497. [CrossRef]

11. Kuyken, B.; Verheyen, P.; Tannouri, P.; Liu, X.; Campenhout, J.V.; Baets, R.; Green, W.M.J.; Roelkens, G. Generation of $3.6 \mu \mathrm{m}$ radiation and telecom-band amplification by four-wave mixing in a silicon waveguide with normal group velocity dispersion. Opt. Lett. 2014, 39, 1349-1352. [CrossRef]

12. Yin, L.; Zhang, J.; Fauchet, P.M.; Agrawal, G.P. Optical switching using nonlinear polarization rotation inside silicon waveguides. Opt. Lett. 2009, 34, 476-478. [CrossRef]

13. Jacome, K.L.; Poulton, C.; Leuthold, J.; Freude, W. Nonlinear silicon-on-insulator waveguides for all-optical signal processing. Opt. Express 2007, 15, 5976-5990.

14. Husko, C.A.; Rossi, A.; Combrie, S.; Tran, Q.V.; Raineri, F.; Wong, C.W. Ultrafast all-optical modulation in GaAs photonic crystal cavities. Appl. Phys. Lett. 2009, 94, 021111. [CrossRef]

15. Colman, P.; Husko, C.; Combrie, S.; Sagnes, I.; Wong, C.W.; de Rossi, A. Temporal solitons and pulse compression in photonic crystal waveguides. Nat. Photonics 2010, 4, 862-868. [CrossRef]

16. Kondo, K.; Baba, T. Dynamic wavelength conversion in copropagating slow-light pulses. Phys. Rev. Lett. 2014, 112, 223904. [CrossRef]

17. Wakabayashi, R.; Fujiwara, M.; Yoshino, K.; Nambu, Y.; Sasaki, M.; Aoki, T. Time-bin entangled photon pair generation from Si micro-ring resonator. Opt. Express 2015, 23, 1103-1113. [CrossRef]

18. Uddin, M.R.; Lingas, N.A.; Nakarmi, B.; Won, Y.H. Waveguide side-wall angle dependant resonance of a Si micro ring-resonato. In Proceedings of the Conference on Lasers and Electro-Optics Pacific Rim Optical Society of America, Busan, Korea, 24-28 August 2015.

19. Guha, B.; Cardenas, J.; Lipson, M. Athermal silicon microring resonators with titanium oxide cladding. Opt. Express 2013, 21, 26557-26563. [CrossRef]

20. Teng, J.; Dumon, P.; Bogaerts, W.; Zhang, H.; Jian, X.; Han, X.; Zhao, M.; Morthier, G.; Baets, R. Athermal Silicon-on-insulator ring resonators by overlaying a polymer cladding on narrowed waveguides. Opt. Express 2009, 17, 14627-14632. [CrossRef]

21. Nguyen, H.C.; Sakai, Y.; Shinkawa, M.; Ishikura, N.; Baba, T. Photonic crystal silicon optical modulators carrier-injection and depletion at $10 \mathrm{~Gb} / \mathrm{s}$. IEEE J. Quantum Electron. 2012, 48, 210-220. [CrossRef]

22. Guan, X.; Wang, X.; Frandsen, L.H. Optical temperature sensor with enhanced sensitivity by employing hybrid waveguides in a silicon Mach-Zehnder interferometer. Opt. Express 2016, 24, 16349-16356. [CrossRef] [PubMed]

23. Klimov, N.N.; Mittal, S.; Berger, M.; Ahmed, Z. On-chip silicon waveguide Bragg grating photonic temperature sensor. Opt. Lett. 2015, 40, 3934-3936. [CrossRef] [PubMed]

24. Furukawa, K.; Ohsuye, K. Effect of culture temperature on a recombinant $\mathrm{CHO}$ cell line producing a Cterminal $\alpha$-amidating enzyme. Cytotechnology 1998, 26, 153-164. [CrossRef] [PubMed]

25. Trenti, A.; Borghi, M.; Biasi, S.; Ghulinyan, M.; Ramiro-Manzano, F.; Pucker, G.; Pavesi, L. Thermo-optic coefficient and nonlinear refractive index of silicon oxynitride waveguides. AIP Adv. 2018, 8, 025311. [CrossRef]

26. Sinclair, G.F.; Tyler, N.A.; Barreto, D.S.J.; Thompson, M.G. Temperature dependence of the Kerr nonlinearity and two-photon absorption in a silicon waveguide at $1.55 \mu \mathrm{m}$. Phys. Rev. Appl. 2019, 11, 044084. [CrossRef]

27. Tanaka, Y.; Tominaka, S.; Kurokawa, T. Precision distance measurement using a twophoton absorption process in a silicon avalanche photodiode with saw-tooth phase modulation. Appl. Opt. 2015, 54, E35-E40. [CrossRef] 
28. Arbabi, A.; Goddard, L.L. Measurements of the refractive indices and thermo-optic coefficients of Si3N4 and SiOx using microring resonances. Opt. Lett. 2013, 38, 3878-3881. [CrossRef]

29. Uenuma, M.; Moooka, T. Temperature-independent silicon waveguide optical filter. Opt. Lett. 2009, 34, 599-601. [CrossRef]

30. Guan, X.; Frandsen, L.H. All-silicon interferometer with multimode waveguides for temperature-insensitive filters and compact biosensors. Opt. Express 2019, 27, 753-760. [CrossRef]

31. Yin, L.; Lin, Q.; Agrawal, G.P. Soliton fission and supercontinuum generation in silicon waveguides. Opt. Lett. 2007, 32, 391-393. [CrossRef]

32. Fleischer, J.W.; Segev, M.; Efremidis, N.K.; Christodoulides, D.N. Observation of two-dimensional discrete solitons in optically-induced nonlinear photonic lattices. Nature 2003, 422, 147-150. [CrossRef] [PubMed]

33. Reid, D.T.; Loza-Alvarez, P.; Brown, C.T.A.; Beddard, T.; Sibbett, W. Amplitude and phase measurement of mid-infrared femtosecond pulses by using cross-correlation frequency-resolved optical gating. Opt. Lett. 2000, 25, 1478-1480. [CrossRef] [PubMed]

34. Parker, D.S.N.; Nunn, A.D.G.; Minns, R.S.; Fielding, H.H. Frequency doubling and fourier domain shaping the output if a femtosecond optical parametric amplifier: Easy access to tuneable femtosecond pulse shapes in the deep ultraviolet. Appl. Phys. B 2009, 94, 181-186. [CrossRef]

35. Liao, J.; Marko, M.; Li, X.; Jia, H.; Liu, J.; Tan, Y.; Yang, J.; Zhang, Y.; Tang, W.; Yu, M.; et al. Cross-correlation frequency-resolved optical gating and dynamics of temporal solitons in silicon nanowire waveguides. Opt. Lett. 2013, 38, 4401-4404. [CrossRef] [PubMed]

36. Nikitin, A.A.; Kondrashov, A.V.; Vitko, V.V.; Ryabcev, L.A.; Zaretskaya, G.A.; Cheplagin, N.A.; Konkin, D.A.; Kokolov, A.A.; Babak, L.I.; Ustinov, A.B.; et al. Carrier-induced optical bistability in the silicon micro-ring resonators undercontinuous wave pumping. Opt. Commun. 2020, 480, 126456. [CrossRef]

Publisher's Note: MDPI stays neutral with regard to jurisdictional claims in published maps and institutional affiliations. 